\title{
Complete synchronization of chaotic atmospheric models by connecting only a subset of state space
}

\author{
P. H. Hiemstra ${ }^{1}$, N. Fujiwara ${ }^{2}$, F. M. Selten ${ }^{1}$, and J. Kurths ${ }^{2}$ \\ ${ }^{1}$ Royal Netherlands Meteorological Institute (KNMI), P.O. Box 201, 3730 AE, De Bilt, The Netherlands \\ ${ }^{2}$ Potsdam Institute for Climate Impact Research (PIK), P.O. Box 6012 03, 14412 Potsdam, Germany \\ Correspondence to: P. H. Hiemstra (paul.hiemstra@knmi.nl)
}

Received: 15 March 2012 - Revised: 11 September 2012 - Accepted: 8 October 2012 - Published: 12 November 2012

\begin{abstract}
Connected chaotic systems can, under some circumstances, synchronize their states with an exchange of matter and energy between the systems. This is the case for toy models like the Lorenz 63, and more complex models. In this study we perform synchronization experiments with two connected quasi-geostrophic (QG) models of the atmosphere with 1449 degrees of freedom. The purpose is to determine whether connecting only a subset of the model state space can still lead to complete synchronization (CS). In addition, we evaluated whether empirical orthogonal functions (EOF) form efficient basis functions for synchronization in order to limit the number of connections. In this paper, we show that only the intermediate spectral wavenumbers (5-12) need to be connected in order to achieve CS. In addition, the minimum connection timescale needed for CS is 7.3 days. Both the connection subset and the connection timescale, or strength, are consistent with the time and spatial scales of the baroclinic instabilities in the model. This is in line with the fact that the baroclinic instabilities are the largest source of divergence between the two connected models. Using the Lorenz 63 model, we show that EOFs are nearly optimal basis functions for synchronization. The QG model results show that the minimum number of EOFs that need to be connected for CS is a factor of three smaller than when connecting the original state variables.
\end{abstract}

\section{Introduction}

Under certain circumstances two chaotic systems can synchronize their state due to coupling (Fujisaka and Yamada, 1983; Pecora and Carroll, 1990; Pikovsky et al., 2003). Synchronization of chaos has found various applications, e.g. secure communications (Pecora et al., 1997). For the atmospheric sciences, chaos synchronization offers interesting possibilities too. Especially in data assimilation, where one of the systems is an atmospheric model, which is connected to another system which are real-world observations (Duane et al., 2006). This form of data assimilation performs well when the models show highly non-linear behavior. In this case other linear data assimilation methods, such as the ensemble Kalman filter (Evensen, 1994), do not perform well (Simon, 2006). As a second example, long-range teleconnections can be seen as synchronization between connected systems within larger atmospheric systems. (Duane, 1997; Duane and Tribbia, 2004). Such teleconnections can also lead to the weaker form of phase synchronization, as shown for ENSO and Indian Monsoon (Maraun and Kurths, 2005; Mokhov et al., 2011). A third example is synchronization in a multi-model approach known as super modeling (van den Berge et al., 2011). Here the ensemble of connected imperfect models synchronize on a common solution that more accurately follows the dynamics of the real system than each of the individual models separately.

An important aspect of chaos synchronization is the amount of exchange of matter and energy between the connected systems. Ideally this exchange of matter and energy should be as limited as possible (Boccaletti et al., 2002). In this study we minimized the exchange of matter and energy by connecting as few state variables as possible. For the super-modeling approach reducing the number of connected variables has the advantage of making the learning of the connection coefficients easier. In addition, connecting less state variables reduces the amount of data (in bytes) that needs to be exchanged between the sub-models. In fullsize weather and climate models this can be a limiting factor. 
The important question is which part of state space needs to be connected in order to achieve synchronization. By synchronization we mean complete synchronization (CS). CS is achieved when the state variables of at least two connected systems are equal and remain equal as the systems evolve in time (Boccaletti et al., 2002).

In addition to limiting the number of connections, we also look at limiting the connection strength, which determines how fast the models converge. This convergence has to counter the tendency of the connected systems to grow apart because of their chaotic nature. Lunkeit (2001) determined the minimum connection strength for a primitiveequations model needed to attain CS. He linked this connection strength to certain processes in the model that are causing a divergence between the two connected models. However, he did not consider limiting the number of connections between the sub-models.

The standard approach is to connect the individual prognostic model variables between models. Alternatively, we propose to connect linear combinations of these model variables. Yang et al. (2006) already showed the potential of this approach by using bred and singular vectors. In this study we use empirical orthogonal functions (EOF) (Preisendorfer and Mobley, 1988). EOFs optimize the description of variance in a given dataset by projecting onto new uncorrelated axes. Earlier studies have successfully used EOFs as basis functions, to reduce the number of degrees of freedom in atmospheric models (Kwasniok, 2007; Ghil et al., 2002; Selten, 1997). Therefore, we hypothesize that the use of EOFs will allow us a similar reduction in the number of connections needed for CS. We define the following main problems:

- Under what circumstances do we achieve complete synchronization (CS)?

- Which minimum subset of the models' state space needs to be connected for CS?

- What is the minimum connecting strength needed for CS?

- Are EOFs efficient basis functions for synchronizing chaotic atmospheric models?

To answer these problems, we analyze two chaotic models: the Lorenz 63 model (Lorenz, 1963) and an intermediate complexity quasi-geostrophic (QG) atmospheric model (Marshall and Molteni, 1993). The Lorenz 63 model is a 3 variable toy model which shows chaotic behavior. It serves as an introduction to connected chaotic systems and the use of EOF analysis to efficiently connect two Lorenz models. The QG model is a much more complex and realistic model with 1449 degrees of freedom, and will serve as a more realistic experiment. The results and methods are presented separately for the Lorenz 63 model and the QG model in Sects. 2 and 3, respectively. The study concludes with a joint discussion of the main problems in the light of our results in Sect. 4.

\section{Part A: Lorenz 63 model}

\subsection{Methods}

\subsubsection{Connected Lorenz 63 model}

In order to see how our method works, we apply this method to a bidirectionally connected Lorenz 63 model (Lorenz, 1963). The equations for the connected system are

$$
\begin{aligned}
& \dot{x}_{1}=\sigma\left(y_{1}-x_{1}\right)+K\left[\boldsymbol{g} \cdot\left(\boldsymbol{x}_{2}-\boldsymbol{x}_{1}+\boldsymbol{\xi}_{1}\right)\right] g_{x} \\
& \dot{y}_{1}=x_{1}\left(\rho-z_{1}\right)-y_{1}+K\left[\boldsymbol{g} \cdot\left(\boldsymbol{x}_{2}-\boldsymbol{x}_{1}+\boldsymbol{\xi}_{1}\right)\right] g_{y} \\
& \dot{z}_{1}=x_{1} y_{1}-\beta z_{1}+K\left[\boldsymbol{g} \cdot\left(\boldsymbol{x}_{2}-\boldsymbol{x}_{1}+\boldsymbol{\xi}_{1}\right)\right] g_{z} \\
& \dot{x}_{2}=\sigma\left(y_{2}-x_{2}\right)+K\left[\boldsymbol{g} \cdot\left(\boldsymbol{x}_{1}-\boldsymbol{x}_{2}+\boldsymbol{\xi}_{2}\right)\right] g_{x} \\
& \dot{y}_{2}=x_{2}\left(\rho-z_{2}\right)-y_{2}+K\left[\boldsymbol{g} \cdot\left(\boldsymbol{x}_{1}-\boldsymbol{x}_{2}+\boldsymbol{\xi}_{2}\right)\right] g_{y} \\
& \dot{z}_{2}=x_{2} y_{2}-\beta z_{2}+K\left[\boldsymbol{g} \cdot\left(\boldsymbol{x}_{1}-\boldsymbol{x}_{2}+\boldsymbol{\xi}_{2}\right)\right] g_{z},
\end{aligned}
$$

with $\sigma=10, \quad \rho=28$, and $\beta=8 / 3$. Here, $g=$ $\left(g_{x}, g_{y}, g_{z}\right)^{T}=(\sin \theta \cos \varphi, \sin \theta \sin \varphi, \cos \theta) \quad$ with $|g|=1$ defines the direction of the connection, $x_{1}=\left(x_{1}, y_{1}, z_{1}\right)^{T}$ and $\boldsymbol{x}_{2}=\left(x_{2}, y_{2}, z_{2}\right)^{T}$ are the state vectors of the systems 1 and 2 , respectively. The noise in the Lorenz systems $\xi_{1}(t)$ and $\xi_{2}(t)$ is independent but obeys the same distribution which is uniform in $[-\sqrt{6}, \sqrt{6}]$. We introduce this noise to prevent the occurrence of complete synchronization. The direction of $\boldsymbol{g}$ is specified with two parameters, $\theta$ and $\varphi$.

It is clear that the synchronizability of $\boldsymbol{x}_{1}$ and $\boldsymbol{x}_{2}$ strongly depends on the connection function $\boldsymbol{g}$ (Huang et al., 2009). Since the direction of $\boldsymbol{g}$ is specified with the two parameters, $\theta$ and $\varphi$, we study the synchronization error $\left\langle\left|\boldsymbol{x}_{1}-\boldsymbol{x}_{2}\right|\right\rangle$ for different $(\theta, \varphi)$, and find the optimal connection function which gives the smallest synchronization error. Here, $\langle\cdot\rangle$ denotes time-average. We introduce the EOFs by measuring the covariance of $\boldsymbol{x}_{1}$, and will check whether the direction of the first EOF corresponding to the largest eigenvalue of the covariance matrix is close to the optimal direction of $\boldsymbol{g}$.

\subsubsection{Covariance matrix and EOFs}

We define the EOFs $\boldsymbol{p}_{i}(i=1,2,3)$ as the eigenvectors of the covariance matrix of model 1 , as defined in the set of equations given in Eq. (1). From a long integration of model 1 , we obtain the covariance matrix $V$ :

$$
V=\frac{1}{N-1}\left(x_{1}-\left\langle x_{1}\right\rangle\right)\left(x_{1}-\left\langle x_{1}\right\rangle\right)^{T} .
$$

From $V$ we can obtain the eigenvectors, i.e. EOFs, and the associate eigenvalues $\lambda_{i}\left(\lambda_{1}>\lambda_{2}>\lambda_{3}\right)$. 


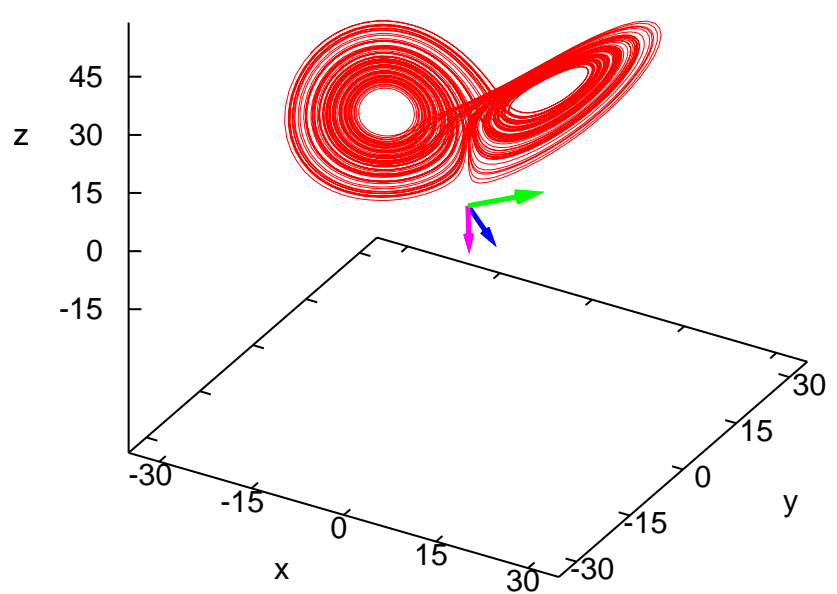

Fig. 1. The Lorenz 63 attractor (red) and its EOFs $\boldsymbol{p}_{1}$ (green), $p_{2}$ (purple), and $\boldsymbol{p}_{3}$ (blue).

\subsection{Results}

\subsubsection{EOFs for the Lorenz 63 model}

The covariance matrix $V$ of model 1 is given by

$V=\left(\begin{array}{ccc}62.80 & 62.80 & 7.6 \times 10^{-4} \\ 62.80 & 81.20 & -1.6 \times 10^{-2} \\ 7.6 \times 10^{-4} & -1.6 \times 10^{-2} & 74.32\end{array}\right)$,

which yield the following EOFs:

$$
\begin{aligned}
& \boldsymbol{p}_{1}=\left(\begin{array}{c}
0.653 \\
0.756 \\
-1.94 \times 10^{-4}
\end{array}\right), \\
& \boldsymbol{p}_{2}=\left(\begin{array}{c}
-2.57 \times 10^{-4} \\
-3.5 \times 10^{-5} \\
-1.00
\end{array}\right), \\
& \boldsymbol{p}_{3}=\left(\begin{array}{c}
0.756 \\
-0.653 \\
-1.71 \times 10^{-4}
\end{array}\right),
\end{aligned}
$$

as the eigenvectors of $V$ corresponding to the eigenvalues $\lambda_{1}=135.47, \lambda_{2}=74.32, \lambda_{3}=8.527$, respectively. In terms of the angles $\theta$ and $\varphi$, they correspond to $\boldsymbol{p}_{1}=(\theta=$ $1.571, \varphi=0.858), \boldsymbol{p}_{2}=(\theta=3.142, \varphi=1.571), \boldsymbol{p}_{3}=(\theta=$ $1.571, \varphi=2.429)$. Note that the sign is arbitrary for an EOF vector, the vector in the opposite direction has the same eigenvalue. So the angles given above are just one of the possible vectors. For example, for $\boldsymbol{p}_{1}$ the second set of angles is equal to $(\theta=1.571, \varphi=4.000)$. Figure 1 shows the attractor of $\boldsymbol{x}_{1}$ and the EOFs $\boldsymbol{p}_{i}$.

\subsubsection{Optimal connection function and EOF}

Here, we show the numerical results of the synchronization error for different $g$ and compare the optimal direction which gives the minimum synchronization error. Moreover, we report the relationship between the optimal direction and the EOF axis.

The synchronization error in the Lorenz 63 model is plotted as a function of $\theta$ and $\varphi$ for different values of the connection strength $K$ (Fig. 2). It gives the relation between vector $g$ and the synchronization error, where a lower error indicates more efficient nudging. Note that we normalized the synchronization error. The normalization consisted of subtracting the mean value for each $K$ value, and dividing by the standard deviations (Becker et al., 1988). We did this to be able to use one color scale for all three $K$ values. As we are primarily interested in the pattern of the synchronization error, this normalization is appropriate.

For large $K$, Fig. 2 shows small synchronization errors at $\theta \approx 1.5, \varphi \approx 1$ and 4, i.e. synchronization is efficient using this vector. This vector matches the first EOF axis $\boldsymbol{p}_{1}$, whose angles are equal to $(\theta=1.571, \varphi=0.858)$ and $(\theta=$ $1.571, \varphi=4000)$. This is confirmed by the cross-section in Fig. 3, which shows that the direction of the EOF vectors (vertical lines) is close to the direction which yields the lowest synchronization error. Therefore, the first EOF $\boldsymbol{p}_{1}$ gives a good approximation of the most efficient connection vector in the Lorenz 63 model. In addition, we find maxima in the synchronization error in the direction of the third EOF axis. This confirms our hypothesis that the third EOF axis is not an efficient means of synchronization.

\section{Part B: Quasi-geostrophic models}

\subsection{Methods}

\subsubsection{Quasi-geostrophic model}

The quasi-geostrophic (QG) model we use in this study provides quite a qualitatively realistic simulation of the atmospheric flow in Northern Hemisphere winter. The model has three levels in the vertical, with pressure as the vertical coordinate. In addition, the model uses a spectral implementation in the horizontal direction:

$q(\lambda, \phi, p, t)=\sum_{n=1}^{21} \sum_{m=-n}^{n} q_{m n}(p, t) Y_{m n}(\lambda, \phi)$

where $\lambda$ is latitude, $\phi$ is longitude, $p$ is pressure (vertical coordinate), $t$ is time, $q_{m n}$ is the spherical harmonic coefficient and $Y_{m n}$ are the spherical harmonic basis functions. The spherical harmonics have been truncated at a total wavenumber $n=21$ (T21).

The QG model is based on the conservation of quasigeostrophic potential vorticity (Marshall and Molteni, 1993), 


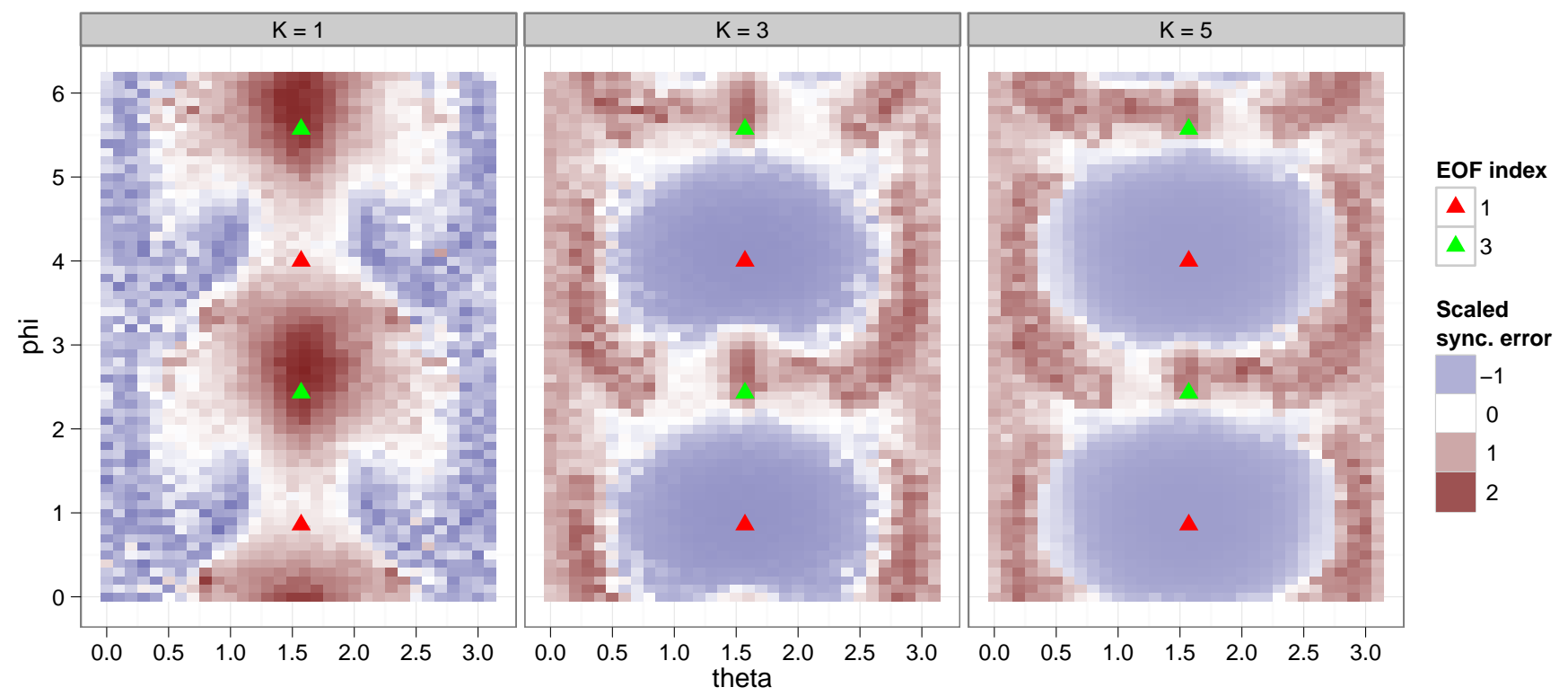

Fig. 2. Plot of the normalized synchronization error $\left\langle\left|x_{1}-x_{2}\right|\right\rangle$ in the $\theta-\varphi$ plane. The red triangles show the $\theta$ and $\varphi$ coordinates of the first EOF axis, $\boldsymbol{p}_{1}$, the green triangles the coordinates of the third EOF axis, $\boldsymbol{p}_{3}$.

and is described by the following set of coupled ordinary differential equations:

$$
\frac{d \boldsymbol{q}}{d t}=F(\boldsymbol{q})
$$

where the non-linear function $F$ describes the advection of potential vorticity, dissipation, forcing and interaction with topography. The $\boldsymbol{q}$ vector contains $n(n+2)=21 \times 23=483$ spherical harmonic coefficients per level, leading to a state space of 1449 dimensions.

\subsubsection{Connecting QG models}

In this study we connect two identical QG models, which have different initial states. The two models are bidirectionally connected:

$\dot{\boldsymbol{q}}_{1}=F\left(\boldsymbol{q}_{1}\right)+C\left(\boldsymbol{q}_{1}-\boldsymbol{q}_{2}\right)$

$\dot{\boldsymbol{q}}_{2}=F\left(\boldsymbol{q}_{2}\right)+C\left(\boldsymbol{q}_{2}-\boldsymbol{q}_{1}\right)$

where $C$ is a $1449 \times 1449$ matrix where the diagonal contains the connection coefficients specifying the strength of the connection $\left(c_{\mathrm{s}}\right)$ for each spherical harmonic. The second term on the right hand side of Eq. (6) is also known as a nudging term. The connection strength $c_{\mathrm{S}}$ is defined as $1 / T_{\mathrm{n}}$, where $T_{\mathrm{n}}$ is the nudging timescale. Expressing the connecting strength as a timescale allows us to compare this timescale to the timescales of processes in the model, e.g. the baroclinic instability. Comparing $T_{\mathrm{n}}$ and other timescales in the model might provide insight into why a certain nudging timescale, i.e. connecting strength, is minimally required.
Instead of connecting all state variables, we can also connect a subset of the spherical harmonics. In this study, we choose to connect a subset of the spectral space by only connecting spherical harmonics above and/or below a certain total wavenumber threshold.

\subsubsection{Connecting using EOFs}

In addition to connecting spherical harmonics with a certain total wave number, we also looked at connecting linear combinations of spherical harmonics instead. We used EOFs to select those linear combinations. EOF analysis exploits the fact that the spherical harmonics do not evolve independently, but in preferred linear combinations. The EOF patterns, or axes, capture these preferred combinations. In other fields of study, EOF analysis is also known as principal component analysis. In this study, we used two different ways to compute the EOFs: (1) calculate EOFs based on normal model output and (2) calculate EOFs based on the synchronization error between two "loosely" connected models. In this case, all spherical harmonical coefficients are connected, but the connection strength is not strong enough to obtain $\mathrm{CS}$ ( $T_{\mathrm{n}}=8$ days). The rationale behind the synchronization error-based EOFs is that the synchronization errors reflect the directions in phase space into which the models most rapidly diverge. Nudging these directions could be an efficient means of obtaining CS. This approach shares some similarities with the work of Yang et al. (2006) on singular and bred vectors.

The EOFs are calculated by performing an eigenvalue decomposition of the covariance matrix $V$ of QG model output. 


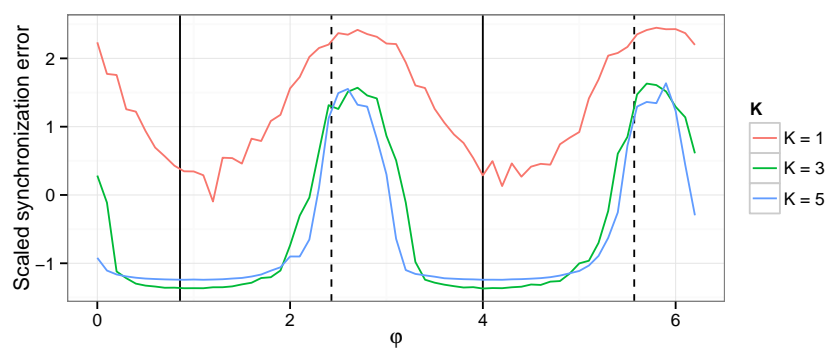

Fig. 3. Cross-section of $\varphi$ versus the scaled synchronization error for several $K$ values at $\theta$ equal to 1.5. The solid vertical lines show the position of the first EOF axis, the dashed vertical line that of the third EOF axis.

The matrix $V$ is constructed by

$V=\frac{1}{N-1}(\Psi-\overline{\mathbf{\Psi}}) M^{2}(\Psi-\overline{\mathbf{\Psi}})^{T}$

where $N$ is the number of model output time steps, $\Psi$ a 1449 $\times N$ matrix of the streamfunction, $\overline{\boldsymbol{\Psi}}$ a 1449 element vector with the sample mean for each spherical harmonic, and $M$ a $1449 \times 1449$ matrix where the diagonal equals 1 for the zonal mode $1(m=0)$ and $\sqrt{2}$ otherwise $(m \neq 0)$. The matrix $M$ is needed because of the way the spherical harmonics are stored in the model. For zonal wavenumbers larger than zero, there are coefficients for both positive and negative zonal numbers, but these are redundant. In our QG model, only the coefficients of the positive zonal numbers are stored. To take into account the contribution of the negative zonal wavenumbers, the matrix $M^{2}$ multiplies the appropriate coefficients by two. In case of calculating normal EOFs, $\Psi$ is the normal QG model output, in case of the synchronization error-based EOFs, $\Psi$ is the difference in the streamfunction between the two connected identical models.

The EOF analysis of a long QG model run resulted in the rotation matrix $E$, where the columns contain the eigenvectors of $V$. This rotation matrix can be used to project state vectors of the streamfunction onto the new EOF axes. After performing the EOF analysis, we construct a new connection matrix $C$ :

$C_{\psi}=E C_{\mathrm{eof}} E^{T} M^{2}$

where $C_{\text {eof }}$ is a diagonal $N_{\text {eof }} \times N_{\text {eof }}$ matrix where the diagonal contains the connection strength, and $N_{\text {eof }}$ the number of EOF axes used for projection. The connection matrix $C_{\psi}$ cannot be directly used in Eq. (6) because the connection is via the potential vorticity $q$. Since $q$ linearly depends on $\psi$, the connection matrix for $q$ is given by

$C_{q}=L C_{\psi} L^{-1}$

where $L$ is a matrix specifying the linear transformation from $\psi$ to $q$, and $q=L \psi$.

\subsubsection{Experimental setup}

For this study we performed three types of experiments:

i. The first experiment is designed to explore the behavior of the synchronization error between two identical bi-directionally connected QG models, as a function of the connection strength and the dimension of the connected linear subspace. Linear subspaces where chosen in two ways. First, by connecting the spherical harmonical coefficients at each level below a total wavenumber threshold $n_{t}$. The number of dimensions associated with $n_{t}$ is given by $N_{\mathrm{sp}}=3 \times n_{t}\left(n_{t}+2\right)$. Raising the threshold incorporates more and more fine scale structures into the connected subspace. The values for $n_{t}$ we explored are 6-14, 16 and 21 , where 21 means that the entire state space is connected. Second, linear subspaces were chosen on the basis of EOFs by connecting only the dominant EOFs below a certain threshold $N_{\text {eof. }}$. We explored the following values for $N_{\text {eof }}: 25,39,50,89,148,178,220,282,382,572$ and 1449 for both the normal and the difference based EOFs. Setting $N_{\text {eof }}=1449$ means that the entire state space is connected. In addition to varying the connected subspace, we also varied the connecting timescale $T_{\mathrm{n}}$, i.e. $T_{\mathrm{n}}=0.5,1,3,5,8,16,32$ days. The models were integrated for 10000 days for every combination of $N_{\text {eof }}$ or $n_{t}$ and $T_{\mathrm{n}}$, leading to a total of $154 \mathrm{QG}$ model runs. Two models where called completely synchronized if the mean synchronization error over the last 5000 days was equal to zero. By zero we mean numerically indistinguishable from zero, i.e. smaller than $10^{-10}$. The first 5000 days where used as a burn in period.

ii. The second experiment is designed to find the minimum $T_{\mathrm{n}}$ value for which we still find CS. For this experiment we fully connect both models, vary $T_{\mathrm{n}}$ and run the model for 36000 days. We estimate the time it takes for two models to synchronize $\left(T_{\mathrm{s}}\right)$ when starting from different initial conditions. More specifically, $T_{\mathrm{S}}$ is defined as the time it takes for the synchronization error to become smaller than $10 \mathrm{e}-10$. We use the graph of $T_{\mathrm{s}}$ versus $T_{\mathrm{n}}$ to estimate the minimum connection strength needed for the models to synchronize.

iii. The third experiment is designed to find the minimum subset of state space that needs to be connected in order to achieve CS. For this experiment we connect the models very strongly, i.e. a $T_{\mathrm{n}}$ of 0.5 days. As in the first experiment, we limit the connection subspace by only connecting a subset of the spherical harmonics or EOFs. For limiting the number of connected spherical harmonics, we not only looked at limiting the connections 
by progressively excluding high wavenumbers, but also by excluding low wavenumbers and by excluding both high and low wavenumbers. In this experiment, we performed a more detailed search for the minimum number of connections needed to get CS. All runs in this experiment consisted of 10000 days. In this experiment we used the same definition of CS as in experiment 1.

\subsubsection{Measure of synchronization}

The degree of synchronization between two QG models is measured by the distance between their states in state space, i.e. the synchronization error. The synchronization error $\left|\psi_{1}-\psi_{2}\right|^{2}$ at time step $t$ is defined as

$\left|\psi_{1}-\psi_{2}\right|^{2}=\left(\psi_{1}-\psi_{2}\right) M^{2}\left(\psi_{1}-\psi_{2}\right)^{T}$

where $\psi_{1}$ and $\psi_{2}$ are 1449 element streamfunction vectors with spherical harmonic coefficients for model 1 and model 2 , respectively. In the experiments we ran the QG models for 10000 days. States are archived four times per day, every $6 \mathrm{~h}$, and the distribution of synchronization error is based on the last 7500 days. The first 2500 days are discarded as a transient.

To quantify the degree of synchronization in relation to a completely unsynchronized state, we propose to scale the synchronization error. We do this by dividing the synchronization error by the time averaged distance between two unconnected models. The values of the scaled synchronization error roughly fall between zero and one, where one implies that the models are totally unsynchronized and $0 \mathrm{im}$ plies CS. We estimated the unsynchronized time averaged distance from a single 10000 day QG model run. We randomly drew 10000 pairs of model states from the available QG model time series and calculated the synchronization error of that pair. The unsynchronized error was estimated as the mean distance of these randomly drawn pairs. Throughout our study, when referring to the synchronization error, we mean the scaled synchronization error.

\subsection{Results}

\subsubsection{EOF analysis}

Figure 4 shows the EOF pattern index versus the cumulative explained variance for both sets of EOFs. We find that the explained variance grows rapidly when including more EOF patterns. To describe $90 \%$ of the variance, we only need 193 normal EOFs and 237 synchronization error-based EOFs.

Spatial patterns of some EOFs are shown in Fig. 5. The first EOF for the normal EOFs shows the greatest variation around a latitude of 50 degrees, which is the location of the jet stream. For synchronization error-based EOFs, the first EOF displays smaller scale structures which have the shape of individual depressions, i.e. baroclinical unstable waves, which are the biggest source of divergence between

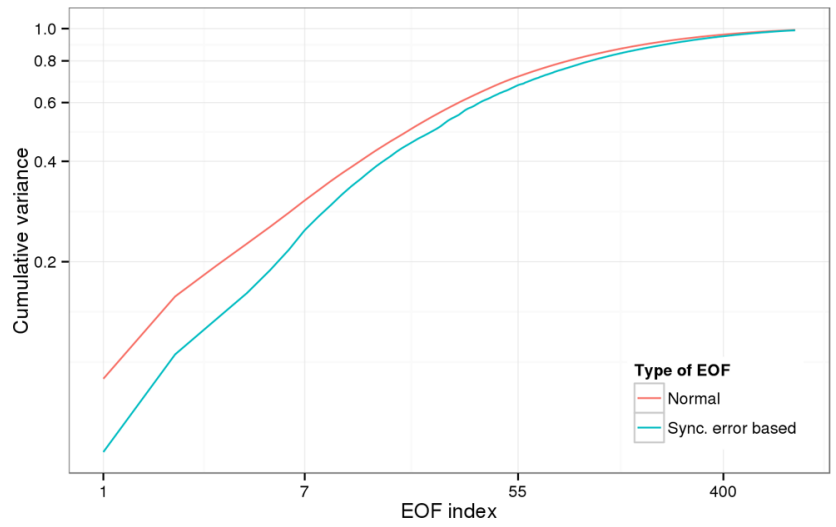

Fig. 4. Cumulative fraction of explained variance of both EOF options versus the number of EOFs. Note that both the $\mathrm{x}$ - and the $\mathrm{y}$ axis are on a $\log$ scale.

the two connected models. In both EOFs options, the Southern Hemisphere ( $\mathrm{SH}$ ) shows almost no variability. This is due to the fact that our QG model was constrained to the winter period on the Northern Hemisphere (NH). In the $\mathrm{NH}$ winter, there is less variability on the $\mathrm{SH}$, where it is summer.

Figure 5 illustrates the patterns in EOFs with a higher index: the patterns have smaller scales and become more noisy. This is the case for both normal and synchronization errorbased EOFs.

\subsubsection{QG model experiments}

\section{Experiment 1}

Figure 5a shows the behavior of the synchronization error when increasing the subspace of state space that is connected, for a number of connection strengths $\left(T_{\mathrm{n}}\right)$. The figure clearly shows that a much smaller subspace needs to be connected when using EOFs instead of spherical harmonics in order to achieve CS. This makes using EOFs a more efficient way of nudging than using spherical harmonics. The performance of the two sets of EOFs is roughly equal, or a bit worse for synchronization based EOFs.

When increasing $T_{\mathrm{n}}$ we see that we need an increasing number of connected state variables to attain CS. Figure 6a suggests that the minimum $T_{\mathrm{n}}$ needed to get CS lies somewhere between 5 and 8 days. Although CS does not occur for higher $T_{\mathrm{n}}$ values, the synchronization error is still smaller than one. This indicates that even a small amount of exchange of matter and energy brings the models closer together than they would be when being unconnected.

EOFs span the attractor more efficiently compared to spherical harmonics and are uncorrelated in time. A relevant question is whether EOF nudging is more efficient only because of this more efficient representation, or if EOFs nudging benefits from some additional dynamical properties. Figure $6 \mathrm{~b}$ explores this by changing the $\mathrm{x}$-axis from the number 
Table 1. Minimum subset of the model state space that needs to be connected for CS in the third experiment $\left(T_{\mathrm{n}}=0.5\right)$.

\begin{tabular}{lllr}
\hline Connection_scheme & subspace & \#variables & variance \\
\hline Normal EOFs & $1-175$ & 175 & 0.89 \\
Sync. error based EOFs & $1-212$ & 212 & 0.89 \\
Connecting intermediate wavenumbers & $5-12$ & 399 & 0.68 \\
Connecting low wavenumbers & $0-11$ & 429 & 0.90 \\
Connecting high wavenumbers & $8-21$ & 1209 & 0.35 \\
\hline
\end{tabular}

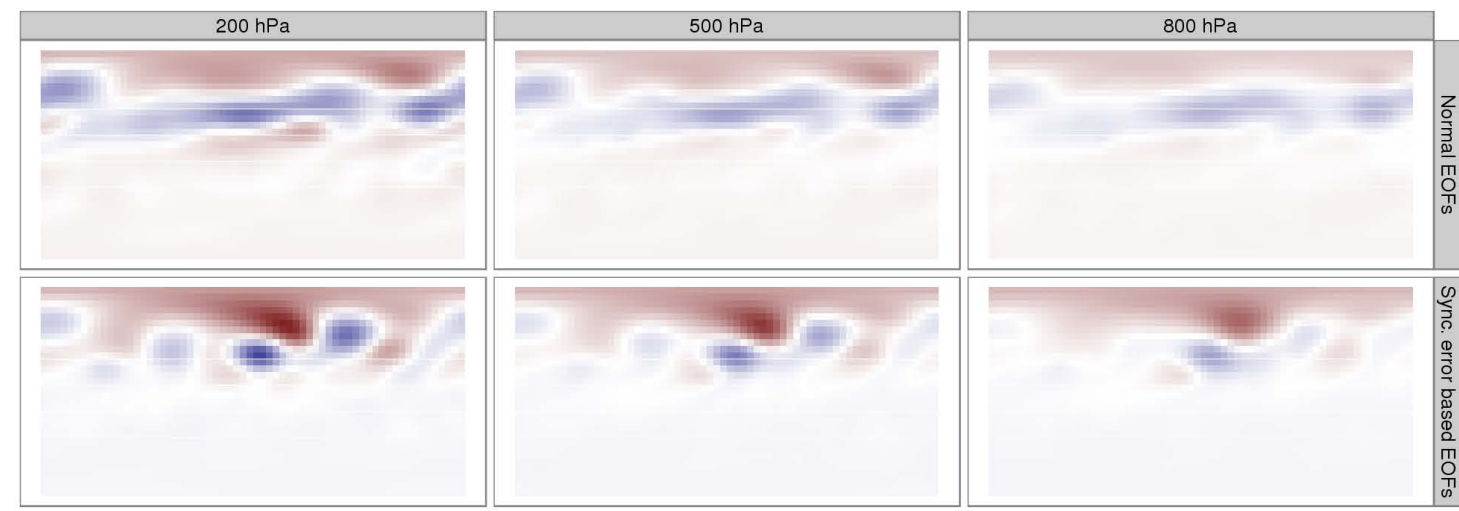

\begin{tabular}{|r|}
\hline psi \\
$-2 e+10$ \\
$-1 e+10$ \\
$0 e+00$ \\
$1 e+10$ \\
$2 e+10$ \\
\hline
\end{tabular}
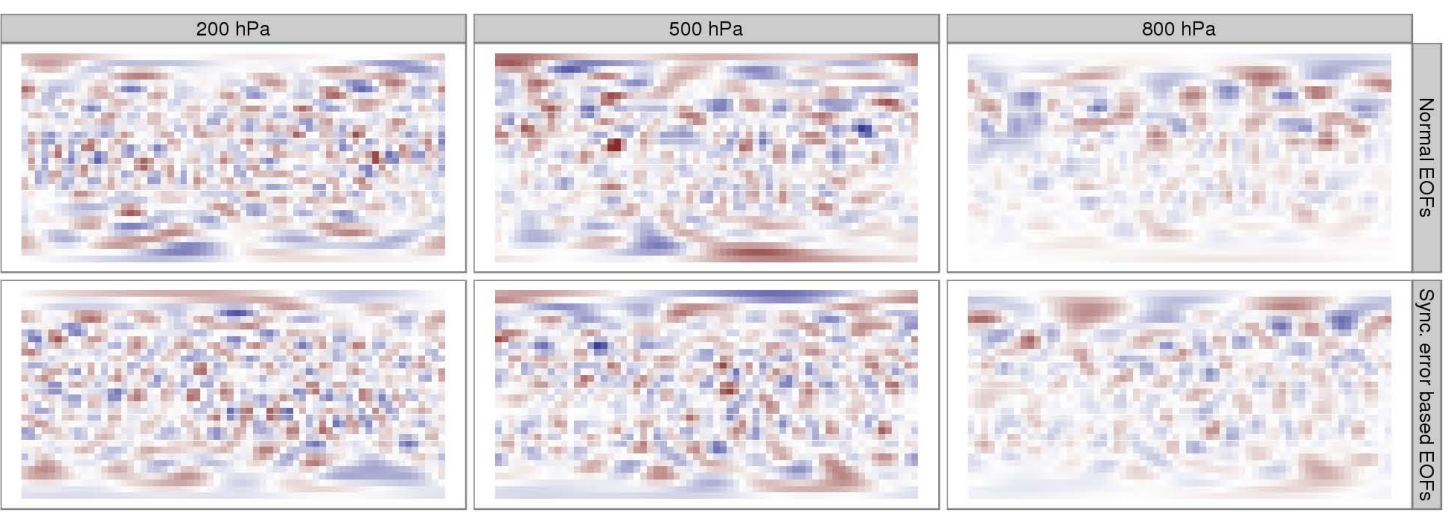

(a)

$\begin{aligned} & \text { psi } \\ &-2 e+10 \\ &-1 e+10 \\ & 0 e+00 \\ & 1 e+10 \\ & 2 e+10\end{aligned}$

Fig. 5. Spatial EOF patterns for (a) the first EOF axis, and (b) for the 572nd EOF axis. The columns in the plots show the three levels in the QG model, the rows in the plot shows the two different types of EOFs: based on normal model output and based on the synchronization error of a loosely connected pair of QG models.

of connected variables to the amount of variance in the connected subspace. The figure suggests that the connected subspace should contain at least $85 \%$ of the variance in order to achieve CS. Moreover, the relation between the synchronization error and the amount of variance in the connected subspace is similar for EOFs and spherical harmonics. This indicates that the reduced number of EOFs needed for CS is merely due to the property of EOFs describing the variance efficiently, and not due to some other dynamical property.

\section{Experiment 2}

In this experiment, we connected the entire subspace and varied the connection strength. To get a more precise estimate of the minimum connection strength required for CS, we plotted $1 / T_{\mathrm{S}}$ versus $1 / T_{\mathrm{n}}$. Here, $T_{\mathrm{S}}$ is the time required for the two models to attain CS. The results are shown in Fig. 7. To get a rough estimate of the minimum nudging time, we fitted a line through these points. The line intersects the $\mathrm{x}$-axis at the nudging strength for which CS is attained in a finite amount of time, i.e. $T_{\mathrm{n}}$ equals 7.3 days.

\section{Experiment 3}

The third experiment finds for a fixed nudging strength, i.e. $T_{\mathrm{n}}=0.5$, the minimum number of EOFs or spherical harmonical wavenumbers that is needed to be connected in order to achieve CS (Table 1). Connecting with normal EOFs proves 


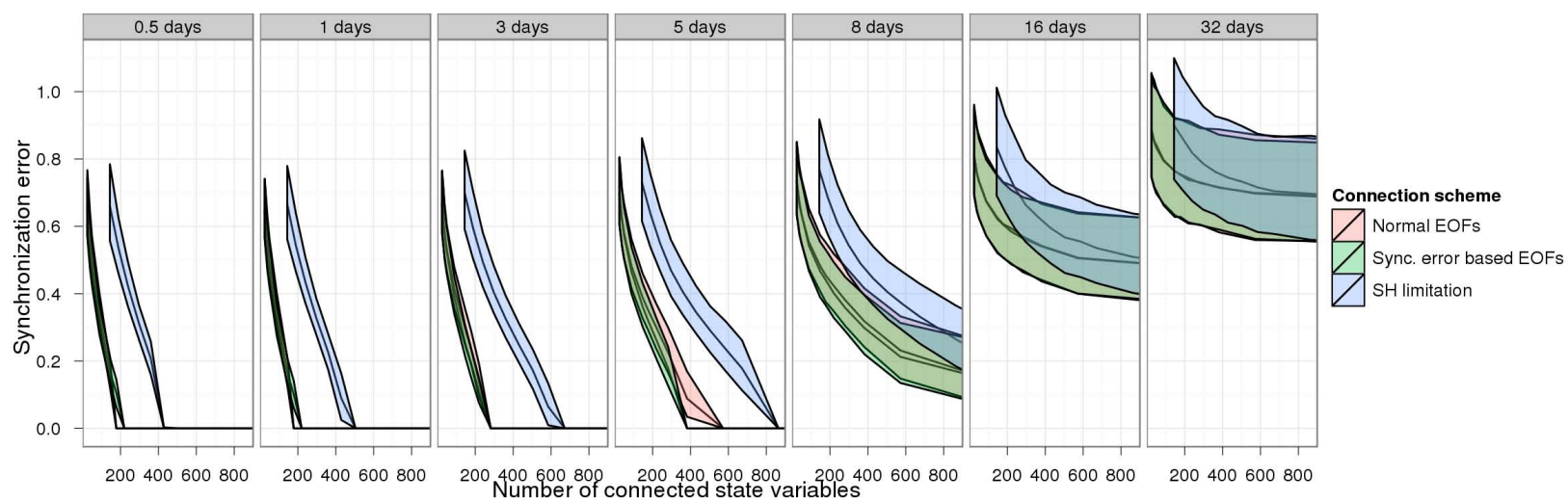

(a)

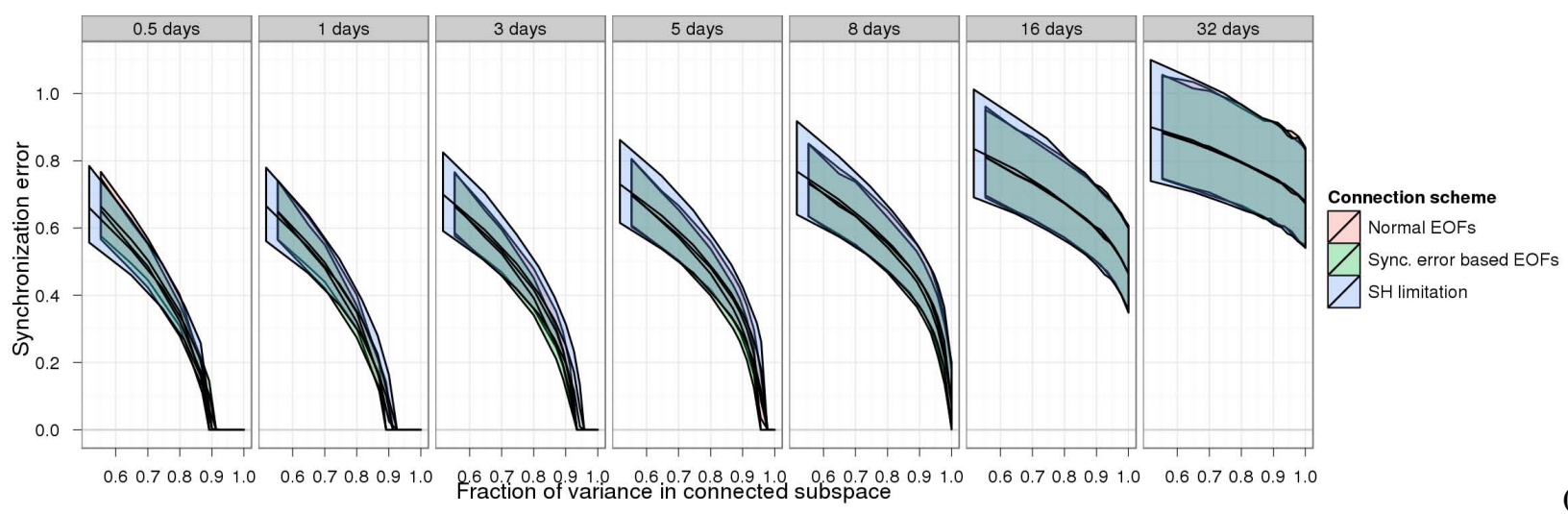

(b)

Fig. 6. (a) Number of state variables connected and (b) variance captured in the connected subspace, versus the scaled synchronization error, i.e. the degree of synchronization, for several $T_{\mathrm{n}}$ values, and for a number of connection schemes. The shaded areas show the $95 \%$ quantile interval. The SH connection scheme works by connecting only the low wavenumbers.

to be most effective in terms of the number of connections. For CS with normal EOFs, we need only 177 connections, which is only $12 \%$ of the total number of state variables. Connecting the submodels using difference based EOFs also performs quite well (15\% of the state variables connected), but less than the normal EOFs. Connecting through spherical harmonics requires a great deal more connected parameters, up to 7 times more. The best result for spherical harmonics was reached by the run which progressively excluded wavenumbers from both the lower and the upper limit. This however still requires roughly twice the amount of connected state variables in comparison to connecting with EOFs. Each of the connection strategies for spherical harmonics requires the wavenumbers between $8-11$ to be connected. These wave numbers are associated with the baroclinically unstable waves in the model.

An alternative to looking at the number of connected state variables is to look at the connected variance. In the base run, we found that the amount of connected variance is roughly equal for both EOFs and spherical harmonics. However, when using other connecting schemes for spherical harmonics, Table 1 suggests that we can get CS with much smaller amounts of connected variance, as low as $35 \%$. Connecting fewer state variables requires more variance in the connected subspace to attain CS.

\section{Discussion and conclusions}

\subsection{Connecting only a subset}

The main purpose of this study was to determine if CS could be achieved by only connecting a subset of the model state space between two bi-directionally connected identical atmosphere models, where only the initial conditions are different. Our results clearly show that this is possible when connecting two QG models. More specifically, our results show that only the intermediate spatial scales need to be connected (total wavenumbers 5-12) in order to attain CS. These results are in line with the work of Duane and Tribbia (2004), who also found that only intermediate scales need to be connected.

Connecting only the large and intermediate scales (total wavenumber 0-11) leads to CS, as does connecting only the intermediate and high scales (total wavenumber 8-21). However, these connection strategies require more connected state variables, and are thus sub-optimal ones. All spherical 


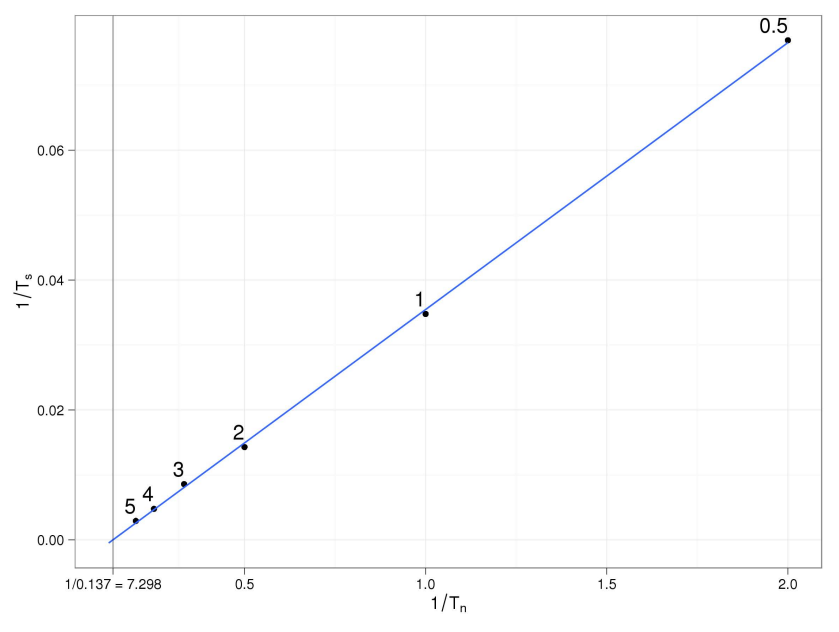

Fig. 7. $1 / T_{\mathrm{S}}$ versus $1 / T_{\mathrm{n}}$ when all variables are connected. The text labels show the associated $T_{\mathrm{n}}$.

harmonical connection strategies connect the intermediate scales (total wavenumber 8-12). We link this to the baroclinically unstable waves in the QG model that cause the growth of flow structures at the scale of these total wavenumbers. As the baroclinically unstable waves are the largest source of divergence between the two models, we find it logical that the connected subset is associated with these unstable waves.

Connecting the submodels through EOFs instead of spherical harmonics also shows that we only need to connect a subset of the model state space in order to obtain CS.

\subsection{Maximum $T_{\mathrm{n}}$}

The maximum value for $T_{\mathrm{n}}$ in order to achieve CS with fully connected models is 7.3 days, further increase of $T_{\mathrm{n}}$ causes the models to no longer synchronize completely. The $T_{\mathrm{n}}$ limit is associated with the timescale of baroclinic instabilities. If $T_{\mathrm{n}}$ becomes larger, these instabilities become more dominant and disrupt CS. Again, it is no surprise that the timescales associated with baroclinically unstable waves play a large role as they are the largest source of divergence between the connected models.

Lunkeit (2001) finds a much larger $T_{\mathrm{S}}$ of around 26 days. We attribute this difference to the fact that the model of Lunkeit (2001) is quite different dynamically from our QG model. Lunkeit (2001) has a stronger jet, and no orography, while the QG model has a realistic winter flow, and correct position and strength of the jet. We find further evidence for the differences by looking at the power spectrum of the $(0,1)$ spherical harmonical coefficient, which represents the global mean relative angular velocity. The spectrum of the QG model, shown in Fig. 8, shows increased variability around the 5-7 day timescale, which coincides with the timescale of the baroclinic instabilities. The spectrum in Lunkeit (2001) shows increased variance in the timescales from 20-50 days. We speculate that this timescale is related

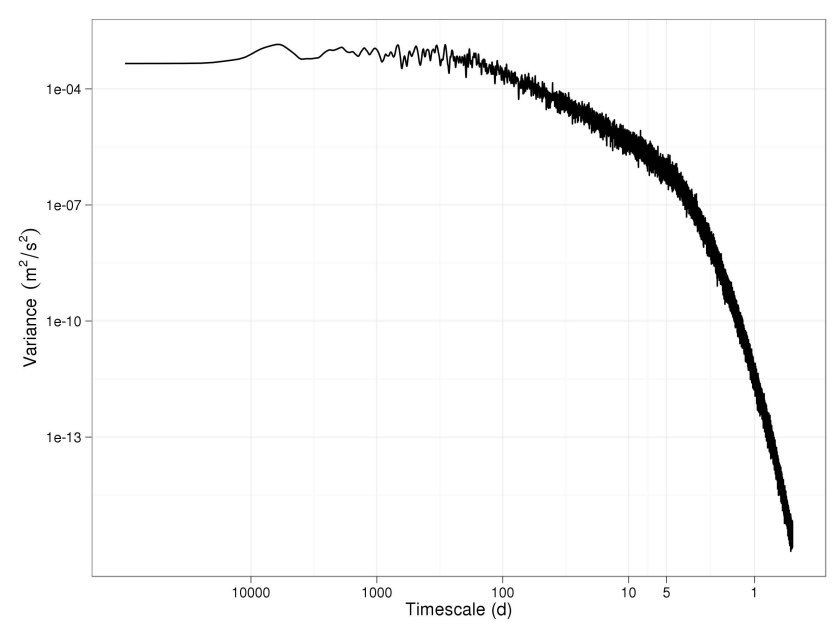

Fig. 8. Power spectrum of the $(0,1)$ spherical harmonical mode.

to the interaction of the baroclinic instabilities with the jet, giving rise to changes in the strength and position of the jet on these timescales.

\subsection{Efficiency of EOFs}

The results in section 2 with the Lorenz model show that, in that case, EOFs are close to the optimal directions for synchronization. Our results for QG models in Sect. 3 show that EOFs are an efficient means to get synchronization, much more efficient than just using the spherical harmonics. However, without performing a brute force analysis like we did for the Lorenz system, we cannot claim that for QG models EOFs are the (almost) optimal connection strategy.

A great number of choices can be made in defining the EOFs, which all influence how effective they are in nudging the QG models to each other. However, in general we expect EOFs to perform better than using the spherical harmonical coefficients. For example, in additional experiment we found that EOFs defined by potential vorticity, and not stream function, are more effective than spherical harmonical coefficients, but less effective than streamfunction based EOFs. We hypothesize that this is caused by the fact that potential vorticity has many more small scale structures. These structures have little effect on the distribution of wind speeds, and thus on advection.

The interpretation of the nudging strength, $T_{\mathrm{n}}$, is not simple when comparing EOF nudging and spherical harmonics nudging. EOF nudging causes a spherical harmonical coefficient to be nudged by a linear combination of all other spherical harmonical coefficients. It is not obvious what $e f-$ fective nudging strength results from this. One might argue that it is not fair to compare the nudging strengths for EOFs and spherical harmonics because of this effective nudging strength. However, when $T_{\mathrm{n}}$ is equal to 0.5 days the nudging strength is effectively infinite, making the issue of the 
effective nudging strength irrelevant. The fact that in this case fewer connections are needed confirms that, although the effective nudging issue might play a role, the main effect remains that EOFs are efficient basis functions for nudging.

An alternative definition of synchronization efficiency could be how much variance is connected between the models. In that case, connecting only the intermediate and small scale spherical harmonics (wavenumber 8-21) is much more efficient than either of the EOF options. However, our goal was to minimize the actual number of connections. Fewer connections makes it easier for learning algorithms to learn how these connections should be defined in a super-modeling approach (van den Berge et al., 2011). In addition, fewer connected state variables reduces the amount of data exchange (in bytes) between the models. Limiting exchange of matter and energy is an important point when applying these technics to full-size climate models. In this case, exchanging too much information can be prohibitively slow.

Of the two EOF alternatives, we expected the EOFs based on synchronization error to perform best. We based our hypothesis on the idea that these EOFs explicitly nudge the directions in phase space were the models diverge most rapidly. Our analysis did not confirm this. EOFs based on differences performed worse than normal EOFs, requiring 212 connected EOFs instead of 175 connected EOFs for the normal EOFs.

We can only speculate why this is the case. One speculation is that transitions between large-scale weather regimes are better described using the normal EOFs. These transitions contribute to a large fraction of the variance, but might contribute less to the day-to-day divergence of nearby trajectories and so are less well captured by the synchronization error-based EOFs. Consequently, during the transitions, the models are more strongly nudged using the normal EOFs and less likely to loose synchronization.

Acknowledgements. This research has been [partially] supported by EU-FET grant SUMO no 266722. N.F. is supported by the Aihara Project, the FIRST program from JSPS, initiated by CSTP. F.M.S. received travel support from DOE Grant \#DE-SC0005238.

All calculation not directly related to the QG model where performed using R (R Development Core Team, 2011; Wickham, 2009, 2011).

Edited by: V. Lucarini

Reviewed by: two anonymous referees

\section{References}

Becker, R., Chambers, J., and Wilks, A.: The new S language, Pacific Grove, Ca.: Wadsworth \& Brooks, 1988, 1, 1-708, 1988.

Boccaletti, S., Kurths, J., Osipov, G., Valladares, D., and Zhou, C.: The synchronization of chaotic systems, Phys. Rep., 366, 1-101, 2002.
Duane, G.: Synchronized chaos in extended systems and meteorological teleconnections, Phys. Rev. E., 56, 6475, doi:10.1103/PhysRevE.56.6475, 1997.

Duane, G. and Tribbia, J.: Weak Atlantic-Pacific teleconnections as synchronized chaos, J. Atmos. Sci., 61, 2149-2168, 2004.

Duane, G. S., Tribbia, J. J., and Weiss, J. B.: Synchronicity in predictive modelling: a new view of data assimilation, Nonlin. Processes Geophys., 13, 601-612, doi:10.5194/npg-13-601-2006, 2006.

Evensen, G.: Sequential data assimilation with a nonlinear quasigeostrophic model using Monte Carlo methods to forecast error statistics, J. Geophys. Res, 99, 143-10, 1994.

Fujisaka, H. and Yamada, T.: Stability theory of synchronized motion in coupled-oscillator systems, Prog. Theor. Phys, 69, 32-47, 1983.

Ghil, M., Allen, M., Dettinger, M., Ide, K., Kondrashov, D., Mann, M., Robertson, A., Saunders, A., Tian, Y., Varadi, F., et al.: Advanced spectral methods for climatic time series, Rev. Geophys, 40, 1003, doi:10.1029/2000RG000092, 2002.

Huang, L., Chen, Q., Lai, Y.-C., and Pecora, L. M.: Generic behavior of master-stability functions in coupled nonlinear dynamical systems, Phys. Rev. E, 80, 036 204, doi:10.1103/PhysRevE.80.036204, http://link.aps.org/doi/ 10.1103/PhysRevE.80.036204, 2009.

Kwasniok, F.: Reduced Atmospheric Models Using Dynamically Motivated Basis Functions, J. Atmos. Sci., 64, 3452-3474, doi:10.1175/JAS4022.1, 2007.

Lorenz, E.: Deterministic non-periodic flow, J. Atmos. Sci., 20, 130-141, 1963.

Lunkeit, F.: Synchronization experiments with an atmospheric global circulation model, Chaos, 11, 47-51, 2001.

Maraun, D. and Kurths, J.: Epochs of phase coherence between El Nino/Southern Oscillation and Indian monsoon, Geophys. Res. Lett., 32, L15709, doi:10.1029/2005GL023225, 2005.

Marshall, J. and Molteni, F.: Toward a dynamical understanding of planetary-scale flow regimes, J. Atmos. Sci., 50, 1792-1818, 1993.

Mokhov, I., Smirnov, D., Nakonechny, P., Kozlenko, S., Seleznev, E., and Kurths, J.: Alternating mutual influence of ElNiño/Southern Oscillation and Indian monsoon, Geophys. Res. Lett, 38, L00F04, doi:10.1029/2010GL045932, 2011.

Pecora, L. and Carroll, T.: Synchronization in chaotic systems, Phys. Rev. Lett., 64, 821-824, 1990.

Pecora, L., Carroll, T., Johnson, G., Mar, D., and Heagy, J.: Fundamentals of synchronization in chaotic systems, concepts, and applications, Chaos, 7, 520, 1997.

Pikovsky, A., Rosenblum, M., and Kurths, J.: Synchronization: A universal concept in nonlinear sciences, 12, Cambridge Univ. Press, 1-432, 2003.

Preisendorfer, R. and Mobley, C.: Principal Component Analysis in Meteorology and Oceanography., New York, pp. 1-455, 1988.

R Development Core Team: R: A Language and Environment for Statistical Computing, R Foundation for Statistical Computing, Vienna, Austria, http://www.R-project.org/, ISBN 3-900051-070,2011 .

Selten, F.: Baroclinic empirical orthogonal functions as basis functions in an atmospheric model, J. Atmos. Sci., 54, 2099-2114, 1997. 
Simon, D.: Optimal state estimation: Kalman, H [infinity] and nonlinear approaches, LibreDigital, 2006.

van den Berge, L. A., Selten, F. M., Wiegerinck, W., and Duane, G. S.: A multi-model ensemble method that combines imperfect models through learning, Earth Syst. Dynam., 2, 161-177, doi:10.5194/esd-2-161-2011, 2011.

Wickham, H.: ggplot2: elegant graphics for data analysis, Springer New York, http://had.co.nz/ggplot2/book, 2009.
Wickham, H.: The Split-Apply-Combine Strategy for Data Analysis, J. Stat. Soft., 40, 1-29, http://www.jstatsoft.org/v40/i01/, 2011.

Yang, S., Baker, D., Li, H., Cordes, K., Huff, M., Nagpal, G., Okereke, E., Villafañe, J., Kalnay, E., and Duane, G.: Data Assimilation as Synchronization of Truth and Model: Experiments with the Three-Variable Lorenz System*, J. Atmos. Sci., 63, 23402354, 2006. 